\title{
Near-Infrared Adaptive Optics Spectroscopy of Binary Brown Dwarfs HD 130948B and HD 130948C
}

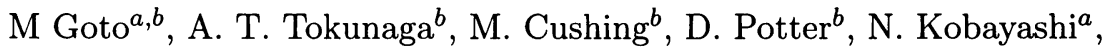 \\ H. Takami ${ }^{a}$, N. Takato ${ }^{a}$, H. Terada ${ }^{a}$, Y. Hayano ${ }^{c}$, M. Iye ${ }^{c}$, \\ W. Gaessler ${ }^{d}$, D. J. Saint-Jacques ${ }^{e}$ \\ ${ }^{a}$ Subaru Telescope, 650 North A'ohoku Place Hilo, HI 96720, USA \\ ${ }^{b}$ Institute for Astronomy, University of Hawaii \\ ${ }^{c}$ National Observatory of Japan at Mitaka \\ ${ }^{d}$ Max-Planck-Institut für Astronomie \\ ${ }^{e}$ University of Montreal
}

\begin{abstract}
We present near-infrared spectroscopy of low-mass companions in the HD 130948 system (Goto et al. 2002a). Adaptive optics on the Subaru Telescope allowed for spectroscopy of the individual components of the 0.13 binary system. Based on a direct comparison with a series of template spectra, we determined the spectral types of HD $130948 \mathrm{~B}$ and C to be $\mathrm{L} 4 \pm 1$. We find they are most likely a binary brown dwarf system.
\end{abstract}

\section{Observation}

HD 130948 is a triple system discovered by Potter et al. (2002a) in the course of an imaging survey for low-mass companions to nearby young stars (See also Potter et al. 2002b in the present proceedings). In order to resolve the close companions, the adaptive optics (AO) system was necessary for the observation.

The spectroscopic observation was made on UT 2001 May 3 using the Infrared Camera and Spectrograph (IRCS; Tokunaga et al. 1998; Kobayashi et al. 2000) with the $8.2 \mathrm{~m}$ Subaru Telescope in conjunction with its AO system (Takami et al. 1998, Gaessler et al. 2001). A medium-resolution grism was used with a $0^{\prime \prime} 10$ slit with the 22 mas pixel scale camera to provide spectra with a resolving power of $800-1000$ in the $H$ and $K$ bands. HD 130948A was used as the reference source for the AO system.

\section{Spectral Type Classification}

We compared the fully reduced spectra of HD 130948B and C with template spectra of low-mass stars. We found none of them match well with the object spectra though the low mass nature is apparent in the strong absorption of $\mathrm{H}_{2} \mathrm{O}$ (Figure 1 left). We concluded that the continuum slope was affected by the AO correction (M. Goto et al. 2002b).

The spectral type was derived in two steps. First, we applied the correction (linear function is assumed) to the spectral slope to maximize the correlation between the template and the observed spectra. Second, the goodness of the 
matches with templates from L1 to L8 were compared. The correlation peaked at L3-L5 in the $H$ band (Figure 1 right) and at L1-L5 in the $K$ band. We derived the spectral type of HD $130498 \mathrm{~B}$ and $\mathrm{C}$ to be $\mathrm{L} 4 \pm 1$, which is consistent with that of L2 \pm 2 determined independently by Potter et al. (2001).

Because the continuum slope is hard to define with AO spectroscopy, the spectral index approach may not work for the classification. We would like to stress the importance to establish a set of individual template stars. The template spectral system should be clean with no binaries and disks, and has at least medium spectral resolution to allow one to calculate the correlation.
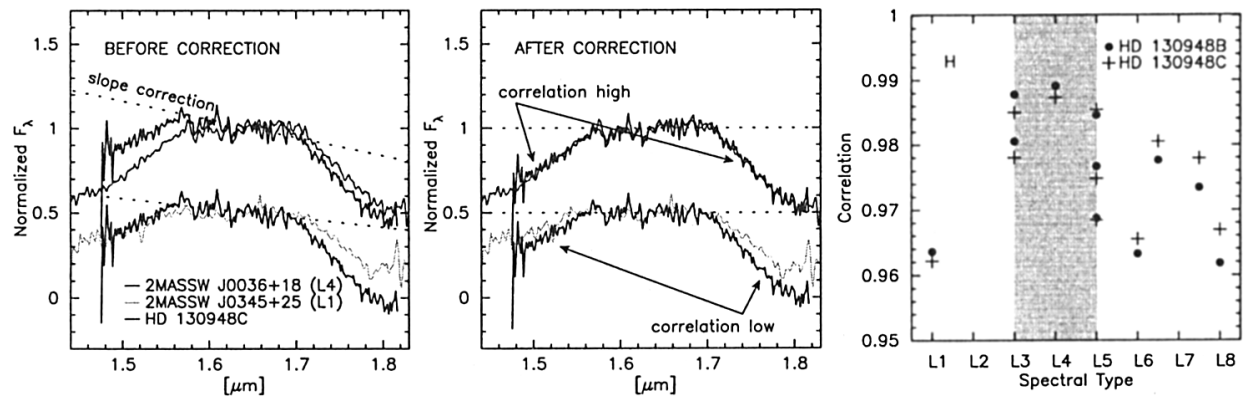

Figure 1. The spectral classification method. The correction of linear functional form is applied. The calculated correlation peaks at L4.

\section{Conversion to Mass}

The range of spectral type was converted to an effective temperature of 1900 $\pm 75 \mathrm{~K}$. On the basis of moderate X-ray activity, a fast rotation period, and the possible membership to the Ursa Major Stream, the age of the primary is estimated to be $0.3-0.8$ Gyr. According to the evolutionary tracks of effective temperatures of low-mass stars calculated by Baraffe et al. (1998), the most likely masses of the objects are $0.040-0.065 \mathrm{M}_{\odot}$.

\section{References}

Baraffe, I., et al. 1998, A\&A, 337, 403

Gaessler, W., et al. 2001, Proc. SPIE, 4494, 30

Goto, M., et al. 2002b, SPIE, 4839, in preparation

Goto, M., et al. 2002a, 567, L59

Kobayashi, N., et al. Proc. 2000, SPIE, 4008, 1056

Potter, D., et al. 2002a, ApJ, 567, L133

Potter, D., et al. 2002b, in this proceeding

Takami, H., et al. 1998, Proc. SPIE, 3353, 500

Tokunaga, A. T., et al. 1998, Proc. SPIE, 3354, 512 